OPEN ACCESS

Edited by:

Alexey Goltsov,

Abertay University, United Kingdom

Reviewed by:

Giovanna Zoccoli,

Università degli Studi di Bologna, Italy

Evgeny Zherebtsov,

Aston University, United Kingdom

*Correspondence:

Zhen-Ni Guo

zhen1ni2@163.com

Yi Yang

doctoryangyi@163.com;

yang_yi@jlu.edu.cn

Specialty section:

This article was submitted to

Vascular Physiology,

a section of the journal

Frontiers in Physiology

Received: 25 July 2018 Accepted: 30 October 2018 Published: 20 November 2018

Citation:

Guo W, Ma H, Liu J, Guo Z-N and Yang Y (2018) Dynamic Cerebral Autoregulation Remains Stable During the Daytime (8a.m. to 8 p.m.) in Healthy Adults. Front. Physiol. 9:1642.

doi: 10.3389/fphys.2018.01642

\section{Dynamic Cerebral Autoregulation Remains Stable During the Daytime (8 a.m. to 8 p.m.) in Healthy Adults}

\author{
Wei-tong Guo ${ }^{1}$, Hongyin $\mathrm{Ma}^{1}$, Jia Liu ${ }^{2}$, Zhen-Ni Guo ${ }^{1,3 *}$ and Yi Yang ${ }^{1,3 *}$ \\ ${ }^{1}$ Department of Neurology, The First Hospital of Jilin University, Changchun, China, ${ }^{2}$ Institute of Advanced Computing and \\ Digital Engineering, Shenzhen Institutes of Advanced Technology, Chinese Academy of Sciences, University Town of \\ Shenzhen, Shenzhen, China, ${ }^{3}$ Department of Neurology, Clinical Trial and Research Center for Stroke, The First Hospital of \\ Jilin University, Changchun, China
}

Many functions of the human body possess a daily rhythm, disruptions of which often lead to disease. Dynamic cerebral autoregulation (dCA) stabilizes the cerebral blood flow to prompt normal neural function. However, whether dCA is stable across the day remains unknown. This study aimed to investigate the daily rhythm of dCA. Fifty-one healthy adults (38.294 \pm 13.279 years, 40 females) were recruited and received six dCA measurements per individual that were conducted at predefined time points: 8:00, 9:00, 11:00, 14:00, 17:00, and 20:00. Although the blood pressure fluctuated significantly, there was no statistical difference in phase difference and gain (autoregulatory parameters) across the six time points. This study demonstrates that dCA remains stable during the interval from 8 a.m. to 8 p.m. and underscores the importance of stable dCA in maintaining cerebral blood flow and neural function.

Keywords: dynamic cerebral autoregulation, blood pressure, cerebral blood flow, daily rhythm, cerebrovascular disease

\section{INTRODUCTION}

Many functions of the human body have their own circadian rhythms and change over the course of a day. Dynamic cerebral autoregulation (dCA) is a process to maintain cerebral blood perfusion at an appropriate state via regulating cerebral vasculature (Budohoski et al., 2013). Cerebral autoregulation typically works with a mean arterial pressure (MAP) in the range of 60 and $150 \mathrm{~mm} \mathrm{Hg}$ in normal conditions (Paulson et al., 1990). Previous studies have shown that patients with hemorrhagic stroke (Ma et al., 2016), ischemic stroke (Guo et al., 2014; Salinet et al., 2018), generalized anxiety (Guo et al., 2018), epilepsy (Lv et al., 2018), and Alzheimer's disease (Shekhar et al., 2017) have impaired dCA. Cerebral autoregulation can be affected many factors, such as $\mathrm{CO}_{2}$ (Meng and Gelb, 2015), nitric oxide (Guo et al., 2016) and altitude level (Jansen et al., 2007). However, the daily rhythm of dCA and whether the time of measurement interferes with the evaluation of dCA are unclear. This study aimed to determine the rhythm of dCA during the interval from 8 a.m. to 8 p.m. in healthy adults.

\section{MATERIAL AND METHODS}

\section{Study Protocol}

The study performed six dCA measurements at six predefined time points: 8:00, 9:00, 11:00, 14:00, 17:00, and 20:00. All the subjects had no history of chronic diseases or acute infections within the 2 weeks before beginning the study. Subjects with intracranial and/or extracranial major vascular 
stenosis/occlusion diagnosed by a transcranial Doppler (EMS9PB, Delica, China) and carotid ultrasound (IU22, Phillips, Andover, MA) were excluded. All the subjects were informed of the study protocol 1 day in advance. Subjects were instructed to refrain from caffeinated drinks and alcohol ingestion for $24 \mathrm{~h}$ before the examination (Claassen et al., 2016). All the subjects were non-smokers. The subjects were asked to avoid strenuous exercise, caffeine, and alcohol during the whole research process. The study was approved by the Ethics Committee of the First Hospital of Jilin University. Written consent was provided by all participants.

\section{DCA Measurement}

Measurements were performed in the specific examination room by a professional technician. The room was quiet and had a controlled temperature of $20^{\circ} \mathrm{C}$ to $24^{\circ} \mathrm{C}$. The subjects were asked to take a relaxed supine position for $10 \mathrm{~min}$. First, the technician measured the arterial blood pressure (ABP) at the brachial artery by an automatic blood pressure monitor (Omron 711, Japan). The continuous ABP was measured non-invasively using a servo-controlled plethysmograph (Finometer Model 1, FMS, Netherlands) at the middle finger. Simultaneously, two 2 $\mathrm{mHz}$ transcranial Doppler probes were placed over the temporal windows to monitor in real-time the bilateral middle cerebral arteries at a depth of $45-60 \mathrm{~mm}$. the probes were fixed with a customized head frame to make sure cerebral blood flow velocity $(\mathrm{CBFV})$ was continuously and stably measured. $\mathrm{CBFV}$ and continuous $\mathrm{ABP}$ were recorded simultaneously from each subject for $10 \mathrm{~min}$. All data were recorded for further assessment and analysis.

\section{Data Analysis}

Data of $\mathrm{ABP}$ and $\mathrm{CBFV}$ were acquired using MATLAB (MathWorks, Inc., US). The dynamic relationship between ABP and CBFV was analyzed by transfer function analysis (TFA) as follows (Claassen et al., 2016). A cross-correlation function between $\mathrm{ABP}$ and $\mathrm{CBFV}$ was used to align the data on in order to eliminate the possible time lags. An anti-alias filter, a third-order Butterworth low-pass filter, with cutoff frequency at $0.5 \mathrm{~Hz}$ was applied so as to down-sample the data to $1 \mathrm{~Hz}$. Welch's method was employed to estimate the autospectrum of ABP, $S_{x x}(f)$, and the cross-spectrum of ABP and CBFV, $S_{x y}(f)$, in frequency domain by averaging the periodograms of the down-sampled $\mathrm{ABP}$ and CBFV with a 50\% overlapped hamming window of $90 \mathrm{~s}$. The transfer function, $H(f)$, was then deviated as:

$$
H(f)=\frac{S_{x y}(f)}{S_{x x}(f)}
$$

Gain and phase difference (PD) can then be calculated from (1) by Equation (2) and (3), respectively, as:

$$
\begin{aligned}
|H(f)| & =\sqrt{\left\{\left|H_{R}(f)\right|^{2}+\left|H_{I}(f)\right|^{2}\right\}}, \\
\theta & =\tan ^{-1}\left[H_{I}(f) / H_{R}(f)\right] .
\end{aligned}
$$

where $\mathrm{R}$ and I denote the real and imaginary parts of the transfer function, respectively. Phase difference (PD), gain, and coherence function within a $0.06-0.12 \mathrm{~Hz}$ frequency range were then derived from TFA to evaluate dCA. A low value of PD indicates that $\mathrm{CBFV}$ follows the changes of $\mathrm{ABP}$ passively, whereas a high value of $\mathrm{PD}$ suggests that $\mathrm{CBFV}$ is actively regulated against the fluctuations of ABP (van Beek et al., 2008). Due to the fact that TFA is a linear model-based method, signals with low coherence between ABP and CBFV $(\leq 0.40)$ were excluded from further statistical analysis.

\section{Statistical Analysis}

The statistical analysis of the data was conducted using IBM SPSS Statistics 24.0 (Armonk, NY, United States), and a twotailed $P$-value $<0.05$ was considered to be statistically significant. The distribution of data was assessed using a one-sample Kolmogorov-Smirnov test. Data are shown as mean \pm standard deviation (SD) for normally distributed continuous variables. Repeated measurement analysis of variance was performed for comparing differences in observed values at different time points.

\section{RESULTS}

The current study enrolled 51 healthy adults $(38.294 \pm 13.279$ years, 40 females). DCA was measured in each subject for six times according to the preset procedure. Thus, the study included 306 records in total for dCA analysis. The coherence of all records was over 0.40 . ABP and heart rate of serial measurements are presented in Table 1. ABP including systolic blood pressure (SBP), diastolic blood pressure (DBP), and mean arterial pressure (MAP) was statistically significant at different time points $(P<$ 0.001 , Figure 1, Table 1). Heart rate remained stable during the whole $12 \mathrm{~h}$ in a day (Figure 1, Table 1). The results of female or male independent analysis and whole subject analysis were consistent (Table 1).

\section{DCA}

From 8:00 to 20:00, the PD was not significantly changed within $12 \mathrm{~h}(P=0.233)$. Furthermore, the PD and gain showed no difference between left and right hemispheres at all the time points $(P=0.573,0.388,0.854,0.263,0.200,0.665)$. Interestingly, the PD tended to be higher at 20:00 compared with the value at 8:00, but it was not statistically significant (Table 1, Figure 1). The gain did not significantly differ among all study time points (Table 1, Figure 1).

\section{DISCUSSION}

The present study investigated the daily rhythm of dCA and the relationship between $\mathrm{ABP}$ and $\mathrm{dCA}$. The major finding of the study was that dCA remains at a stable level during the daytime. Furthermore, dCA did not fluctuate following the changes of ABP.

Clinically, dCA measurement can correctly reflect most aspects of the autoregulatory response (Tiecks et al., 1995). Previous studies have shown that dCA was affected by the sympathetic nerve (Hamner et al., 2010), cholinergic nerve (Hamner et al., 2012), myogenic mechanisms (Tan et al., 2013), and metabolic control (Payne, 2018). Although these 


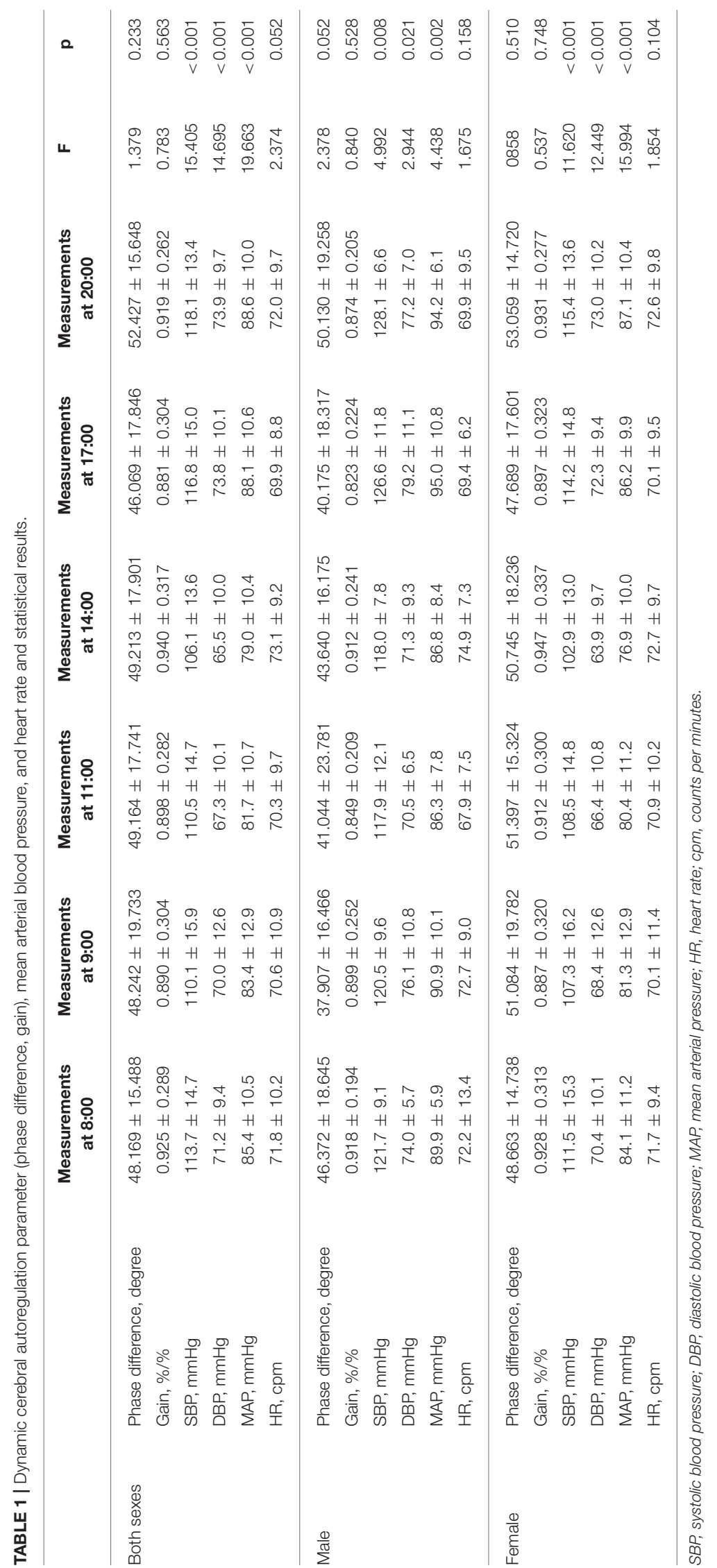


A
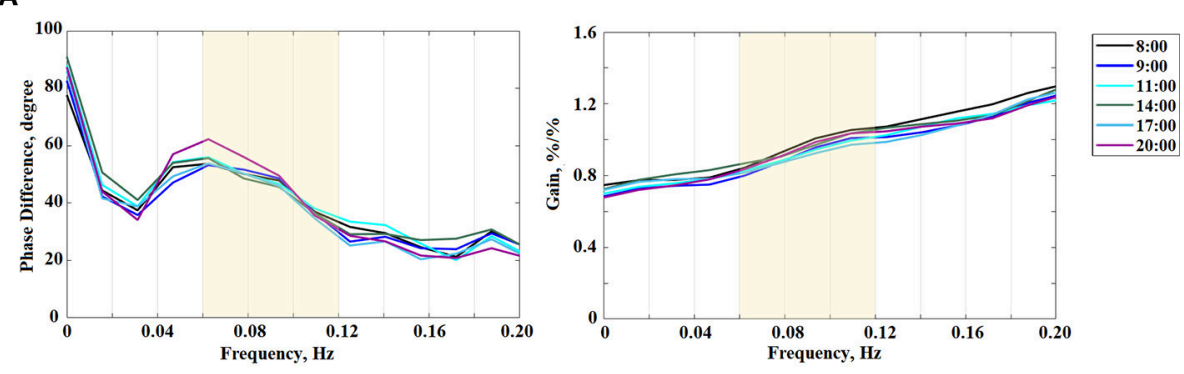

B
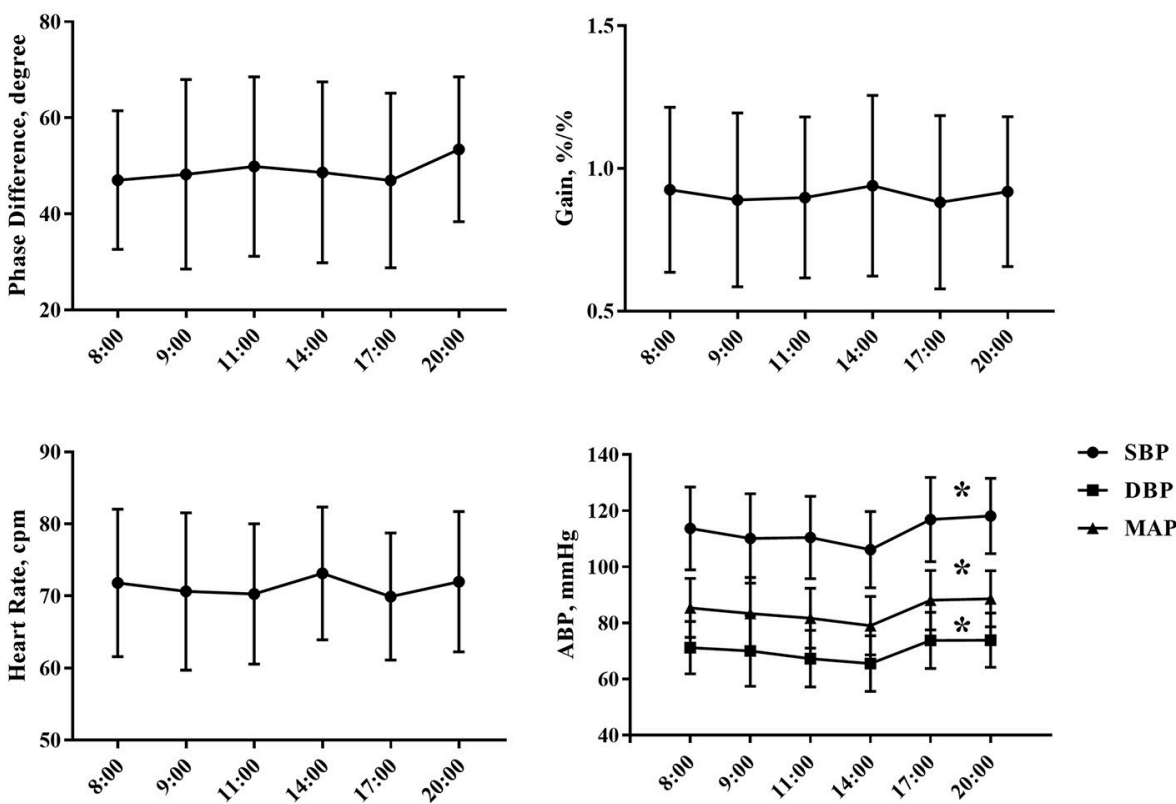

FIGURE 1 | (A) The autoregulatory parameter (phase difference and gain) derived from the transfer function analysis and estimated over a range (0.06-0.12 $\mathrm{Hz}$, the yellow area) at 8:00, 9:00, 11:00, 14:00,17:00, and 20:00. Each colored line denotes the averaged PD or gain estimated from all subjects at one particular time point that we collected the data. (B) Statistical analysis of serial dCA, arterial blood pressure, and heart rate of serial measurements. The solid point represents means, and the whiskers denote standard deviation. ${ }^{\star} P<0.05$ for comparison of different time points by repeated measurement analysis of variance. HR, indicates heart rate; cpm, counts per minutes; SBP, systolic blood pressure; DBP, diastolic blood pressure; MAP, mean arterial pressure.

mechanisms have their own change rules, we showed that dCA is maintained at a stable level. The present study may indicate that the daily rhythm of dCA is independent of the change rule of one single mechanism.

Cerebral autoregulation functions to stabilize cerebral blood flow and metabolism while the ABP changes (Lassen, 1959). When dCA was impaired, the cerebral blood flow would change with fluctuations in blood pressure, leading to cerebrovascular disease. ABP fluctuation has been found to be a potent risk factor for cerebrovascular disease (Yano and Kario, 2012). Due to the morning blood pressure surge, most strokes occur in the morning hours (Marler et al., 1989; Yano and Kario, 2012). Similar to previous studies (Atkinson et al., 2010; Douma and Gumz, 2018), the present study reproduced the finding of ABP fluctuations. However, we found that dCA was independent of $\mathrm{ABP}$ fluctuations during the daytime.

Our analysis indicated that healthy adults have stable and functional dCA to maintain brain perfusion, similar to the findings of a previous study (Brys et al., 2003). Chi et al have demonstrated that dCA was interchangeable and effective by assessing from the internal carotid artery compared with that from the middle cerebral artery (Chi et al., 2017). Also, dCA assessed by using a $5 \mathrm{~min}$ recording was identical with that using a $10 \mathrm{~min}$ recording in the clinical application (Chi et al., 2018). Despite the stability of different recording time and different measuring locations, we have discovered the stable rhythm of dCA during the 8:00-20:00 interval. And, conversely to the abundant literature showing differences in cerebral blood flow between sexes (Barnes, 2017) and the influence of sex on circadian rhythms (Yan and Silver, 2016), the present study has shown the same results for both sexes.

In the literature, dCA was reported to be reduced at 6 a.m. -8 a.m. (Ainslie et al., 2007), which is different from the present study. We speculate that the differences might arise from three reasons: (1) different detection time of cerebral autoregulation: Ainslie et al performed the morning dCA 
measurement in the early morning (6a.m. -8 a.m.), while our study performed the morning dCA measurement at $8 \mathrm{AM}$. Because we don't know the differences between "early morning" and "morning" of the dCA, we cannot exclude the difference caused by the detection time points; (2) the sex of the subjects: Ainslie et al recruited all male subjects, while the present study recruited both male and female subjects; (3) the age of the subjects; Ainslie et al recruited young subjects with a mean age of 25 . However, our study recruited middle-aged subjects with a mean age of 38 .

The present study shows that $\mathrm{dCA}$ is a reliable indicator and remains at a stable level during the daytime and was not affected by fluctuations of ABP. Thus, given its stability and reliability, it possible to assist in diagnosing and assessing the cerebrovascular function of cerebrovascular diseases, generalized anxiety, epilepsy and Alzheimer's disease. Simultaneously, the $\mathrm{dCA}$ also can be used to evaluate the treatment effect of the above disease.

However, there are several limitations to the study. The current study lacks the nocturnal and early morning rhythm of $\mathrm{dCA}$. The main reason is that monitoring $\mathrm{dCA}$ requires the subjects to stay awake. If night monitoring is carried out, subjects may not sleep well, which may affect daytime results. Furthermore, due to the nature of the observational study, we did not collect the blood samples at different time points to test the changes of biomarkers. These questions should be pursued in future studies.

\section{REFERENCES}

Ainslie, P. N., Murrell, C., Peebles, K., Swart, M., Skinner, M. A., Williams, M. J., et al. (2007). Early morning impairment in cerebral autoregulation and cerebrovascular $\mathrm{CO}_{2}$ reactivity in healthy humans: relation to endothelial function. Exp. Physiol. 92, 769-777. doi: 10.1113/expphysiol.2006.036814

Atkinson, G., Jones, H., and Ainslie, P. N. (2010). Circadian variation in the circulatory responses to exercise: relevance to the morning peaks in strokes and cardiac events. Eur. J. Appl. Physiol. 108, 15-29. doi: $10.1007 / \mathrm{s} 00421-009-1243-y$

Barnes, J. N. (2017). Sex-specific factors regulating pressure and flow. Exp. Physiol. 102, 1385-1392. doi: 10.1113/ep086531

Brys, M., Brown, C. M., Marthol, H., Franta, R., and Hilz, M. J. (2003). Dynamic cerebral autoregulation remains stable during physical challenge in healthy persons. Am. J. Physiol. Heart Circ. Physiol. 285, H1048-1054. doi: 10.1152/ajpheart.00062.2003

Budohoski, K. P., Czosnyka, M., Kirkpatrick, P. J., Smielewski, P., Steiner, L. A., and Pickard, J. D. (2013). Clinical relevance of cerebral autoregulation following subarachnoid haemorrhage. Nat. Rev. Neurol. 9, 152-163. doi: 10.1038/nrneurol.2013.11.

Chi, N. F., Ku, H. L., Wang, C. Y., Liu, Y., Chan, L., Lin, Y. C., et al. (2017). Dynamic cerebral autoregulation assessment using extracranial internal carotid artery doppler ultrasonography. Ultrasound Med. Biol. 43, 1307-1313. doi: 10.1016/j.ultrasmedbio.2017.02.003

Chi, N. F., Wang, C. Y., Chan, L., and Hu, H. H. (2018). Comparing different recording lengths of dynamic cerebral autoregulation: 5 versus 10 Minutes. BioMed Res. Int. 2018:7803426. doi: 10.1155/2018/7803426

Claassen, J. A., Meel-van den Abeelen, A. S., Simpson, D. M., and Panerai, R. B. (2016). Transfer function analysis of dynamic cerebral autoregulation: a white paper from the International Cerebral Autoregulation Research Network. J. Cereb. Blood Flow Metab. 36, 665-680. doi: 10.1177/0271678x15626425
In summary, the rhythm of dCA keep was steady during the 8 a.m. -8 a.m. interval in healthy adults, and it is not influenced by the fluctuations of $\mathrm{ABP}$. For evaluating $\mathrm{dCA}$, one random measurement of $\mathrm{dCA}$ is reliable.

\section{AUTHOR CONTRIBUTIONS}

WG design the study, acquired the data, analyzed and interpreted of data, drafted the manuscript. Z-NG analyzed and interpreted of data and revised the manuscript. JL analyzed and interpreted of data and obtained funding. HM acquired the data and revised the manuscript. YY studied concept and designed the study, critical revised the manuscript, supervised the study and obtained funding.

\section{FUNDING}

This article was supported by the National Key R\&D Program of China (2016YFC1301600), JLUSTIRT (2017TD-12), and Jilin provincial development and reform commission (2017C018) to YY, and National Natural Science Foundation of China (Grant NO. 81871447) to JL.

\section{ACKNOWLEDGMENTS}

We thanked all the volunteers for their contributions to the study.

Douma, L. G., and Gumz, M. L. (2018). Circadian clock-mediated regulation of blood pressure. Free Radic. Biol. Med. 119, 108-114. doi: 10.1016/j.freeradbiomed.2017.11.024

Guo, Z. N., Liu, J., Xing, Y., Yan, S., Lv, C., Jin, H., et al. (2014). Dynamic cerebral autoregulation is heterogeneous in different subtypes of acute ischemic stroke. PLoS ONE 9:e93213. doi: 10.1371/journal.pone.0093213

Guo, Z. N., Lv, S., Liu, J., Wang, Z., Jin, H., Qiu, Q., et al. (2018). Compromised dynamic cerebral autoregulation in patients with generalized anxiety disorder: a study using transfer function analysis. BMC Psychiatry 18:164. doi: 10.1186/s12888-018-1713-Z

Guo, Z. N., Shao, A., Tong, L. S., Sun, W., Liu, J., and Yang, Y. (2016). The role of nitric oxide and sympathetic control in cerebral autoregulation in the setting of subarachnoid hemorrhage and traumatic brain injury. Mol. Neurobiol. 53, 3606-3615. doi: 10.1007/s12035-015-9308-x

Hamner, J. W., Tan, C. O., Lee, K., Cohen, M. A., and Taylor, J. A. (2010). Sympathetic control of the cerebral vasculature in humans. Stroke 41, 102-109. doi: 10.1161/strokeaha.109.557132

Hamner, J. W., Tan, C. O., Tzeng, Y. C., and Taylor, J. A. (2012). Cholinergic control of the cerebral vasculature in humans. J. Physiol. 590, 6343-6352. doi: 10.1113/jphysiol.2012.245100

Jansen, G. F., Krins, A., Basnyat, B., Odoom, J. A., and Ince, C. (2007). Role of the altitude level on cerebral autoregulation in residents at high altitude. J. Appl. Physiol. 103, 518-523. doi: 10.1152/japplphysiol.01429.2006

Lassen, N. A. (1959). Cerebral blood flow and oxygen consumption in man. Physiol. Rev. 39, 183-238. doi: 10.1152/physrev.1959.39.2.183

Lv, S., Guo, Z. N., Jin, H., Sun, X., and Jia, M. (2018). Compromised dynamic cerebral autoregulation in patients with Epilepsy 2018:6958476. doi: 10.1155/2018/6958476

Ma, H., Guo, Z. N., Liu, J., Xing, Y., Zhao, R., and Yang, Y. (2016). Temporal course of dynamic cerebral autoregulation in patients with intracerebral hemorrhage. Stroke 47, 674-681. doi: 10.1161/strokeaha.115.011453 
Marler, J. R., Price, T. R., Clark, G. L., Muller, J. E., Robertson, T., Mohr, J. P., et al. (1989). Morning increase in onset of ischemic stroke. Stroke 20, 473-476.

Meng, L., and Gelb, A. W. (2015). Regulation of cerebral autoregulation by carbon dioxide. Anesthesiology 122, 196-205. doi: 10.1097/aln.0000000000 000506

Paulson, O. B., Strandgaard, S., and Edvinsson, L. (1990). Cerebral autoregulation. Cerebrovasc. Brain Metab. Rev. 2, 161-192.

Payne, S. J. (2018). Identifying the myogenic and metabolic components of cerebral autoregulation. Med. Eng. Phys. doi: 10.1016/j.medengphy.2018.04.018

Salinet, A. S., Silva, N. C., Caldas, J., de Azevedo, D. S., de-Lima-Oliveira, M., Nogueira, R. C., et al. (2018). Impaired cerebral autoregulation and neurovascular coupling in middle cerebral artery stroke: influence of severity? 271678x18794835. doi: 10.1177/0271678x18794835.

Shekhar, S., Wang, S., Mims, P. N., Gonzalez-Fernandez, E., Zhang, C., He, X., et al. (2017). Impaired cerebral autoregulation-a common neurovascular pathway in diabetes may play a critical role in diabetes-related alzheimer's disease. Curr. Res. Diabetes Obes. J. 2:555587.

Tan, C. O., Hamner, J. W., and Taylor, J. A. (2013). The role of myogenic mechanisms in human cerebrovascular regulation. J. Physiol. 591, 5095-5105. doi: 10.1113/jphysiol.2013.259747

Tiecks, F. P., Lam, A. M., Aaslid, R., and Newell, D. W. (1995). Comparison of static and dynamic cerebral autoregulation measurements. Stroke 26, 1014-1019. van Beek, A. H., Claassen, J. A., Rikkert, M. G., and Jansen, R. W. (2008) Cerebral autoregulation: an overview of current concepts and methodology with special focus on the elderly. J. Cereb. Blood Flow Metab. 28, 1071-1085. doi: $10.1038 /$ jcbfm. 2008.13

Yan, L., and Silver, R. (2016). Neuroendocrine underpinnings of sex differences in circadian timing systems. J. Steroid Biochem. Mol. Biol. 160, 118-126. doi: 10.1016/j.jsbmb.2015.10.007

Yano, Y., and Kario, K. (2012). Nocturnal blood pressure, morning blood pressure surge, and cerebrovascular events. Curr. Hypertens Rep. 14, 219-227. doi: 10.1007/s11906-012-0261-z.

Conflict of Interest Statement: The authors declare that the research was conducted in the absence of any commercial or financial relationships that could be construed as a potential conflict of interest.

Copyright (๑) 2018 Guo, Ma, Liu, Guo and Yang. This is an open-access article distributed under the terms of the Creative Commons Attribution License (CC BY). The use, distribution or reproduction in other forums is permitted, provided the original author(s) and the copyright owner(s) are credited and that the original publication in this journal is cited, in accordance with accepted academic practice. No use, distribution or reproduction is permitted which does not comply with these terms. 